Tohoku J. Exp. Med., 2008, 214, 159-163

\title{
Allogeneic Peripheral Blood Stem Cell Transplantation from Related Donors Mismatched at 2 HLA Loci in the Host-versus-Graft Direction
}

\author{
Toshiniko Ando, ${ }^{1}$ Toshiaki Yujiri, ${ }^{1}$ Jun Nomiyama, ${ }^{1}$ Noriyuki Mitani, ${ }^{1}$ \\ Masato Seguchi, ${ }^{1}$ Atsushi Matsubara ${ }^{1}$ and Yukio Tanizawa ${ }^{1}$ \\ ${ }^{1}$ Third Department of Internal Medicine, Yamaguchi University School of Medicine, Ube, Japan
}

In allogeneic stem cell transplantation, immune reactions can occur in 2 directions. The recipient's lymphocytes can recognize the donor's cells as "foreign" and attempt to kill them, which results in the host-versus-graft (HVG) reaction that is commonly termed graft rejection. The other direction is the graft-versus-host $(\mathrm{GVH})$ reaction. When the recipient is homozygous at a mismatched human leukocyte antigen (HLA) locus, HLA disparity is present only in the former direction and not in the latter direction. If transplants harvested from such an HVG-mismatched donor can be used to achieve stable engraftment with minimal toxicity, then these donors can potentially be a useful alternative donor source. Here, we report 2 patients ( 1 with acute myeloblastic leukemia and another with lymphoblastic lymphoma) who were transplanted with peripheral blood stem cells (PBSCs) obtained from related donors mismatched at 2 HLA loci in the HVG direction but completely matched in the GVH direction. Our conditioning regimen, consisting of busulfan, cyclophosphamide, low-dose total body irradiation (TBI) (4 Gy), and fludarabine, achieved successful engraftment with an acceptable level of regimen-related toxicity. Our experience suggests that PBSC transplantation with an HVG-mismatched related donor and an appropriate conditioning regimen may be a therapeutic option for patients in whom early transplantation is desirable. - hematopoietic stem cell transplantation; graft failure; host-versus-graft mismatch; total-body irradiation; fludarabine.

Tohoku J. Exp. Med., 2008, 214 (2), 159-163.

(C) 2008 Tohoku University Medical Press

The engraftment of donor stem cells during the course of stem cell transplantation (SCT) consistently results in host-versus-graft (HVG) tolerance. The degree of donor-recipient histoincompatibility is considered the most important risk factor that prevents successful engraftment. It has been reported that graft failure occurs in approximately $20 \%$ of patients transplanted with stem cells with mismatches at 2 human leukocyte anti- gen (HLA) loci (Anasetti et al. 1989). The incidence of graft rejection increases with the degree of HLA disparity between the donor and recipient. When the recipient is homozygous at a mismatched HLA locus, HLA disparity is present only in the direction of rejection and not in the direction of graft-versus-host disease (GVHD). Therefore, recipients who are homozygous at 1 or more mismatched loci were found to have a sig-

Received January 11, 2007; revision accepted for publication January 18, 2008.

Correspondence: Toshiaki Yujiri, M.D., Ph.D., Third Department of Internal Medicine, Yamaguchi University

School of Medicine, 1-1-1 Minamikogushi, Ube, Yamaguchi 755-8505, Japan.

e-mail: yujirit@yamaguchi-u.ac.jp 
nificantly higher incidence of graft failure than that seen in heterozygous recipients (Forman et al. 1994). Increased immunosuppression by using intensified preparative regimens has improved the rate of successful engraftment of haploidentical marrow stem cells with mismatches at 2-3 HLA loci (Anasetti et al. 1989). A decrease in the incidence of graft failure from $17 \%$ to $9 \%$ was reported when the hyperfractionated total body irradiation (TBI) dose was increased from 12 Gy to 13.2 or 14.4 Gy over 6 days (11-12 doses of 120 cGy t.i.d.) (Thomas et al. 1999). However, the intensive conditioning regimen resulted in increased toxicity and the risk of infectious complications after transplantation.

To our knowledge, few systematic reports on SCT with mismatches at more than 2 loci in the HVG direction and at less than 1 locus in the $\mathrm{GVH}$ direction. If stable engraftment can be achieved under such conditions, SCT may theoretically be performed with a lower risk of GVHD than that entailed in haploidentical SCT. This approach might also increase the number of eligible donors. Here, we report 2 patients who were successfully transplanted with peripheral blood stem cells (PBSCs) from related donors mismatched at 2 loci in the HVG direction (based on serological typing of HLA-A, HLA-B, and HLADR antigens of the donor and recipient). The transplantation was accomplished by employing a conditioning regimen consisting of busulfan, cyclophosphamide, low-dose TBI (4 Gy), and fludarabine. Both patients achieved early engraftment with an acceptable level of toxicity.

\section{Clinical Findings}

\section{Patient 1}

Acute myeloblastic leukemia with trilineage dysplasia (AML with TLD) was diagnosed in a 27 years old woman with complaints of general fatigue. Complete remission (CR) could not be achieved by induction therapy with idarubicin and cytarabine. High-dose cytarabine therapy induced $\mathrm{CR}$, but the patient suffered from severe sepsis during the neutropenic period. The deletion of chromosome 7, TLD, and the requirement of 2 courses of induction therapy for achieving CR were all suggestive of a poor prognosis. The patient had no HLA-matched related donor; further, it was not feasible to wait for an unrelated donor. We therefore performed allogeneic PBSC transplantation (allo-PBSCT) with a graft obtained from her sister (mismatched at 2 loci in the HVG direction but completely matched in the GVH direction). The ethics committee of our institution granted approval, and written informed consent was obtained from the donor and the recipient. The patient was homozygous for both the HLA-A and HLA-B loci (Table 1). In order to prevent graft failure, we referred to the published recommended dosages of $\mathrm{CD} 34^{+}$cells for $\mathrm{T}$ celldepleted transplantation in patients at high risk for rejection (Champlin et al. 2002). Accordingly, we set a target of $5 \times 10^{6} \mathrm{CD} 34^{+}$cells $/ \mathrm{kg}$ body weight of the recipient. The conditioning regimen consisted of 4 Gy TBI (in 2 fractions), $16 \mathrm{mg} / \mathrm{kg}$ oral busulfan, $120 \mathrm{mg} / \mathrm{kg}$ cyclophosphamide, and 180 $\mathrm{mg} / \mathrm{m}^{2}$ fludarabine. GVHD prophylaxis comprised $3 \mathrm{mg} / \mathrm{kg}$ cyclosporine A and short-term

TABLE 1. Serological HLA typing of patients and donors.

\begin{tabular}{|c|c|c|c|c|c|c|}
\hline Patient 1 & \multicolumn{2}{|c|}{ HLA-A } & \multicolumn{2}{|c|}{ HLA-B } & \multicolumn{2}{|c|}{ HLA-DR } \\
\hline Patient & 24 & - & 52 & - & 4 & 15 \\
\hline Donor & 24 & 26 & 52 & 54 & 4 & 15 \\
\hline Patient 2 & \multicolumn{2}{|c|}{ HLA-A } & \multicolumn{2}{|c|}{ HLA-B } & \multicolumn{2}{|c|}{ HLA-DR } \\
\hline Patient & 24 & - & 7 & - & 1 & - \\
\hline Donor & 24 & - & 54 & 7 & 4 & 1 \\
\hline
\end{tabular}


methotrexate therapy. The number of $\mathrm{CD} 34^{+}$cells actually transplanted was nearly equal to the target at $4.8 \times 10^{6}$ cells $/ \mathrm{kg}$ body weight. Granulocyte engraftment was defined as the successful transplantation of $>0.5 \times 10^{9}$ cells $/ 1$ on day 11 . Platelet engraftment was defined as the successful transplantation of $20 \times 10^{9}$ cells/l over 3 days without the requirement of transfusion prior to day 11. Chimerism analysis performed using short tandem repeat analysis of peripheral blood mononuclear cells on day 24 showed that the graft was completely of the donor type at this time. The only non-hematological organ dysfunction observed was grade III stomatitis. From day 0 to day 28, Regimen-related toxicity was graded according to the National Cancer Institute Common Toxicity Criteria (NCI-CTC) version 2.0. The complications encountered were acute GVHD (skin stage 2, grade I), early-onset bronchiolitis obliterans organizing pneumonia (BOOP; approximately day +50 ), extensive chronic GVHD, and hemorrhagic cystitis caused by adenovirus type 11 . However, the patient achieved stable engraftment and remained in CR for 50 months after transplantation.

\section{Patient 2}

Lymphoblastic lymphoma was diagnosed in a 52 years old woman complaining of general fatigue, fever, and right supraclavicular lymphadenopathy. She was treated with combination chemotherapy (mitoxantrone, etoposide, cytarabine, etc.), and CR was achieved; however, relapse occurred. Reinduction chemotherapy was instituted, and CR was achieved again. The patient had no HLA-matched related donor, and it was not feasible to wait for an unrelated donor. Therefore, allo-PBSCT was performed using stem cells from her nephew (mismatched at 2 loci in the HVG direction and completely matched in the GVH direction) (Table 1). The patient and donor signed an approval consent form. The conditioning regimen consisted of 4 Gy TBI (in 2 fractions), $8 \mathrm{mg} / \mathrm{kg}$ oral busulfan, $120 \mathrm{mg} / \mathrm{kg}$ cyclophosphamide, and $180 \mathrm{mg} / \mathrm{m}^{2}$ fludarabine. GVHD prophylaxis comprised $3 \mathrm{mg} / \mathrm{kg}$ cyclosporine $\mathrm{A}$ and short-term methotrexate therapy. The dose of $\mathrm{CD} 34^{+}$cells was $10.8 \times 10^{6}$ cells $/ \mathrm{kg}$ body weight of the recipient. Granulocyte engraftment was achieved on day 10 after transplantation. Platelet engraftment was achieved on day 13. A chimerism analysis of bone marrow cells performed using XY fluorescence in situ hybridization (FISH) on day 23 and short tandem repeat assessment of peripheral blood mononuclear cells on day 44 indicated that the cells were completely of the donor type. The only non-hematological organ dysfunction observed was grade III stomatitis. The complications included acute GVHD (skin stage 2, gut stage 1, grade II), cytomegalovirus antigenemia, and extensive chronic GVHD. This patient has achieved stable engraftment and CR for 45 months.

\section{Discussion}

HLA incompatibility, recipient sensitization, patient diagnosis, conditioning regimen, posttransplantation immune suppression, T-cell depletion, and hematopoietic stem cell dose are all considered risk factors for graft failure (Thomas et al. 1999). Recipients homozygous at 1 or more mismatched loci showed a significantly higher incidence of graft failure than that seen in heterozygous recipients (Forman et al. 1994). Recently, Ottinger et al. (2003) reported that transplantation from partially HLA-matched related donors with more than a single HLA class I mismatch in the HVG direction was associated with a high risk of graft failure. The rate of graft failure increased significantly with the number of HLA class I disparities: from 6 in $125(4.8 \%)$ and 1 in 28 (3.6\%) patients with no mismatch and 1 mismatch, respectively, to 3 in $22(13.6 \%)$ and 3 in $6(50 \%)$ patients with 2 and 3 mismatches, respectively. However, these results were based on bone marrow transplantation (BMT) and not PBSCT.

Beelen et al. (1997) reported a prospective comparison of the results obtained after the allogeneic transplantation of unmanipulated recombinant human granulocyte colony-stimulating factor (rhG-CSF)-mobilized PBSCs from HLAgenotypically identical sibling donors or alternative family donors. Of 24 patients, 23 who had alternative family donors exhibited at least 1 
major histocompatibility complex (MHC) class I or II antigen disparity in the HVG direction. However, all these patients achieved timely and sustained engraftment without episodes of primary or secondary graft failure. To our knowledge, there have been no reports regarding engraftmentfailure rates in allogeneic PBSCT in which antigen disparity in the HVG direction is greater than that in the GVH direction.

Increased immunosuppression by intensified preparative regimens has to a certain extent improved the rate of successful engraftment of haploidentical marrow stem cells with 2-3 mismatches in HLA loci (Anasetti et al. 1989). However, such intensive conditioning regimens result in increased toxicity and risk of infectious complications after transplantation. Antibodies directed against host $\mathrm{T}$ cells to prevent the rejection of mismatched hematopoietic stem cells have been used as an alternative to an increased TBI dose. The addition of antithymocyte globulin (ATG) $(10 \mathrm{mg} / \mathrm{kg} /$ day, from day -2 to day +3$)$ to the standard preparative regimen of cyclophosphamide (120 mg/kg) plus TBI (15.75 Gy) was used for grafts mismatched in the HVG direction. However, in this setting, ATG failed to decrease the incidence of graft failure. Conditioning regimens, including an increased TBI dose and/or ATG addition, were considered very rigorous in these cases because Patient 1 had only recently recovered from severe sepsis, and Patient 2 was a relatively elderly patient. In recent years, fludarabine-based transplantation using a reduced-intensity conditioning regimen has been developed and has proven to be capable of achieving stable engraftment with minimal toxicity (Slavin et al. 1998). Therefore, instead of increasing the TBI dose, we added fludarabine to the conditioning regimen. We retained a TBI dose of $4 \mathrm{~Gy}$ in the conditioning regimen based on the reduced-intensity conditioning regimens published for cord blood transplantation (Miyakoshi et al. 2004) and transplantation from unrelated donors for aplastic anemia (Deeg et al. 2001). Although our conditioning regimen was myeloablative, the associated toxicity was comparatively mild, and both patients achieved stable engraftment.
Because the present report relates to only 2 patients, it is premature to draw generalized conclusions concerning the therapeutic potential of this type of conditioning regimen. However, if the procedure of transplantation from an HVGmismatched donor is established, these type of donors might be a useful alternative donor source when early transplantation is desirable.

\section{References}

Anasetti, C., Amos, D., Beatty, P.G., Appelbaum, F.R., Bensinger, W., Buckner, C.D., Clift, R., Doney, K., Martin, P.J., Mickelson, E., Nisperos, B., O'Quigley, J., Ramberg, R., Sanders, J.E., Stewart, P., Storb, R., Sullivan, K.M., Witherspoon, R.P., Thomas, E.D. \& Hansen, J.A. (1989) Effect of HLA compatibility on engraftment of bone marrow transplants in patients with leukemia or lymphoma. $N$. Engl. J. Med., 320, 197-204.

Beelen, D.W., Ottinger, H.D., Elmaagacli, A., Scheulen, B., Basu, O., Kremens, B., Havers, W., Grosse-Wilde, H., Ulrich, W. \& Schaefer, U.W. (1997) Transplantation of filgrastim-mobilized peripheral blood stem cells from HLAidentical sibling or alternative family donors in patients with hematologic malignancies: a prospective comparison on clinical outcome, immune reconstitution, and hematopoietic chimerism. Blood, $\mathbf{9 0}, 4724-4735$.

Champlin, R., Hesdorffer, C., Lowenberg, B., Martelli, M.F., Mertelsmann, R.H., Reisner, Y. \& Schmitz, N. (2002) Haploidentical 'megadose' stem cell transplantation in acute leukemia: recommendations for a protocol agreed upon at the Perugia and Chicago meetings. Leukemia, 16, 427-428.

Deeg, H.J., Amylon, M.D., Harris, R.E., Collins, R., Beatty, P.G., Feig, S., Ramsay, N., Territo, M., Khan, S.P., Pamphilon, D., Leis, J.F., Burdach, S., Anasetti, C., Hackman, R., Storer, B. \& Mueller, B. (2001) Marrow transplants from unrelated donors for patients with aplastic anemia: minimum effective dose of total body irradiation. Biol. Blood Marrow Transplant., 7, 208-215.

Forman, S.J., Blume, K.G. \& Thomas, E.D. (1994) Bone Marrow Transplantation. Blackwell Scientific Publications, Boston.

Miyakoshi, S., Yuji, K., Kami, M., Kusumi, E., Kishi, Y., Kobayashi, K., Murashige, N., Hamaki, T., Kim, S.W., Ueyama, J., Mori, S., Morinaga, S., Muto, Y., Masuo, S., Kanemaru, M., Hayashi, T., Takaue, Y. \& Taniguchi, S. (2004) Successful engraftment after reduced-intensity umbilical cord blood transplantation for adult patients with advanced hematological diseases. Clin. Cancer Res., 10, 3586-3592.

Ottinger, H.D., Ferencik, S., Beelen, D.W., Lindemann, M., Peceny, R., Elmaagacli, A.H., Husing, J. \& Grosse-Wilde, H. (2003) Hematopoietic stem cell transplantation: contrasting the outcome of transplantations from HLA-identical siblings, partially HLA-mismatched related donors, and HLA-matched unrelated donors. Blood, 102, 1131-1137.

Slavin, S., Nagler, A., Naparstek, E., Kapelushnik, Y., Aker, M., Cividalli, G., Varadi, G., Kirschbaum, M., Ackerstein, A., Samuel, S., Amar, A., Brautbar, C., Ben-Tal, O., Eldor, A. \& Or, R. (1998) Nonmyeloablative stem cell transplantation and cell therapy as an alternative to conventional bone 
marrow transplantation with lethal cytoreduction for the treatment of malignant and nonmalignant hematologic diseases. Blood, 91, 765-763.
Thomas, E.D., Forman, S.J. \& Blume, K.G. (1999) Hematopoietic Cell Transplantation 2nd ed., Blackwell Scientific Publications, Boston. 